\title{
The Fracture Behavior and Mechanical Properties of a Support Structure for Additive Manufacturing of Ti-6Al-4V
}

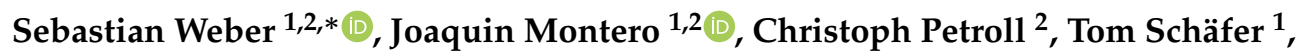 \\ Matthias Bleckmann ${ }^{2}$ and Kristin Paetzold ${ }^{1}$ \\ 1 Institute for Technical Product Development, University of the Bundeswehr Munich, \\ Werner-Heisenberg-Weg 39, 85579 Neubiberg, Germany; j.montero@unibw.de (J.M.); \\ tom.schaefer@unibw.de (T.S.); kristin.paetzold@unibw.de (K.P.) \\ 2 Bundeswehr Research Institute for Materials, Fuels and Lubricants (WIWeB), Institutsweg 1, \\ 85435 Erding, Germany; christophpetroll@bundeswehr.org (C.P.); \\ matthiasbleckmann@bundeswehr.org (M.B.) \\ * Correspondence: s.weber@unibw.de; Tel.: +49-89-6004-3685
}

Received: 1 April 2020; Accepted: 24 April 2020; Published: 27 April 2020

\begin{abstract}
In the laser powder bed fusion processes for metal additive manufacturing, a support structure is needed to fix the part to the base plate and to support overhanging regions. Currently the importance of support structure for a successful build process is often underestimated and some effects are not yet well understood. Therefore, this study investigates the fracture behavior and mechanical properties of thin additive manufactured struts using the titanium alloy Ti-6Al-4V and specific machine parameters for support structures. Tensile tests were performed for different strut diameters and the fracture surfaces were analyzed using a laser microscope and a scanning electron microscope. Additionally, the porosity was examined with micro-CT scans. The results were compared with a different set of parameters used for solid parts. The experiments revealed that struts produced with support parameters had no significantly lower tensile strength than the comparative parts. Despite that, some porosity and around two percent of defects on the fracture surface for parts using the solid parameter set have been found. Parts with support parameters show no porosity, even though the energy density is around 30\% lower compared to the solid parameter set.
\end{abstract}

Keywords: support structure; additive manufacturing; Powder Bed Fusion; titanium alloy; Ti-6Al-4V; fracture behavior; mechanical properties

\section{Introduction}

Additive manufacturing (AM) is a process for the rapid creation of complex parts in small batches. It is associated with a small number of design restrictions and near net-shaped products. Metal additive manufacturing is a manufacturing process with which to create a part based on 3D model data by subsequently adding material layer upon layer [1]. This is in contradiction to subtractive manufacturing methods, which create the final product through material removal usually from a semi-finished part. For machines used in the consumer sector, 3D Printing is typically used as a synonym to AM. Nowadays its applications range from rapid prototyping $(R P)$, rapid tooling $(R T)$ and rapid manufacturing $(R M)$ to fully functional parts and even simple assemblies [2]. Based on the ability of AM to create complex shapes, it is usually related to the idea of design freedom. Nevertheless some restrictions depending on the used material and process apply [3]. The most common AM technology for industrial metal parts and therefore the focus of this article is laser powder bed fusion (L-PBF). 
L-PBF uses a laser as an energy source. L-PBF is described as an "AM process in which thermal energy selectively fuses regions of a powder bed" [1].

In L-PBF powder particles of a powder bed are selectively fused together by the laser beam, merging them to a single layer of a part. The beam is guided in the horizontal plane based on trajectories defined through the slicing of a 3D model, which is the method for dividing a 3D model into a finite number of equally spaced layers. After each layer is finished, the build plate is lowered by a fixed amount specifying the layer height. New powder is then placed on top of the previous layer by a recoating device. Repeating this procedure, the part is build layer upon layer. To avoid oxidation, the build chamber is evacuated of remaining oxygen by a constant flow of an inert gas; for instance, argon.

Due to high melting temperatures of the titanium alloy and the extremely high cool down rates, the material tends to expand and contract, leading to high residual stresses. Additionally, during solidification there is not enough time for the microstructure to solidify in an equilibrium phase and thereby resolve the stressed state. These internal stresses lead to severe distortions and deformation of the part [4]. To prevent build failures based on large deformations, a support structure is added for even heat dissipation and to anchor the part on the build plate [5]. After the AM process is finished, these support structures need to be mechanically removed in a post-processing step.

A support structure has different tasks to fulfill in metal additive manufacturing. On the one hand, it is needed to create an evenly distributed heat gradient from the part to the base plate, and on the other hand, deformations need to be reduced or prevented by anchoring the part to the base plate. Another important role is the support of overhanging structures [6], which would otherwise sink down into the loose powder bed. There are different types of support structures used in metal additive manufacturing. Among the most common are block-support, point-support, web-support, contour-support and line-support [6,7]. These types can be implemented in a simple manner with state of the art pre-processing software and are sometimes even generated automatically. More complex approaches use the stacking of unit cells, which is then called a lattice support structure [8,9]. Lately investigations were made with heat and topology optimization, resulting in promising tree-like structures consisting of stems with multiple branches each $[10,11]$. The benefits of those support structures are the more flexible design and lesser powder consumption, and therefore, build time is reduced when compared to traditional structures. Therefore tree-like support is part of this ongoing research. For this study, the focus is set exclusively on the stem and hence on test samples consisting of multiple struts.

A general rule for a support structure is to use only as much as necessary and as little as possible, due to the fact that an increase in support volume leads directly to longer print times and a higher powder consumption. On the other hand, insufficient support leads to failed print jobs. Knowing the fracture properties of supports and estimating residual stresses in an early stage are key elements for primary support structure design; i.e., for a support that is added during the part design. Due to the current underestimation of the importance and the design of the support structure, the process can fail as a result of severe warping and/or collapse of the part or support. Typically, a support structure is built using manufacturer-provided parameters differing from those for structural parts. Consequently, a thorough investigation of the mechanical and fracture behavior is needed for a proper design. The resulting mechanical properties of the support structures are also very important for structural simulations that lead to new support designs.

Previous research investigated the mechanical properties and fracture behaviors of additive manufactured titanium samples with geometry according to standards $[12,13]$ or other large cross-sectional areas [14], often together with examination of the microstructure $[15,16]$. Therefore the influences of a small diameter are not taken into account. On the other hand, a lot of effort is put into the design of support structures. Their optimal geometry is analyzed using structural or thermal simulations [7,11], or parameter optimizations are performed [6]. 
This study aims to fill the gap between support structure design and investigations of mechanical properties for finished parts, to further understand the in-process failure of supports. Therefore, tensile test samples with thin struts, representing the supports, were additively manufactured using the titanium alloy Ti-6Al-4V. Specific machine parameters for support structure were used. Tensile tests were carried out for different strut diameters and build parameters. The resulting fracture surfaces were examined with a scanning electron microscope. To distinguish between pores and trapped particles, additional micro-CT scans were performed. All results were compared with a set of parameters for structural solid parts.

\section{Materials and Methods}

\subsection{Titanium Alloy Powder-Ti-6Al-4V}

All test samples used in this study were produced using the titanium alloy powder Ti64ELI-A $L M F$ from the manufacturer Trumpf, where ELI (extra low interstitials) stands for improved ductility and better fracture toughness. The titanium alloy is also known under the designation Ti-6Al-4V and is one of the most common materials used in metal additive manufacturing [6]. It was produced using plasma atomization, resulting in particle sizes between $15 \mu \mathrm{m}$ and $45 \mu \mathrm{m}$. The particle size distribution is $D 10=20.6 \mu \mathrm{m}, D 50=33.5 \mu \mathrm{m}$ and $D 90=43.0 \mu \mathrm{m}$ [17]. The chemical composition of the powder is shown in Table 1.

Table 1. Chemical powder composition [17].

\begin{tabular}{ccccccccc}
\hline & Al & V & Fe & O & C & N & H & Ti \\
\hline Min. & 5.50 & 3.50 & $\leq 0.25$ & $\leq 0.13$ & $\leq 0.08$ & $\leq 0.05$ & $\leq 0.012$ & residual \\
Max. & 6.50 & 4.50 & $\leq 0.019$ & 0.09 & 0.01 & 0.02 & 0.0025 & balance \\
Measured & 6.49 & 3.99 & 0.19 & 0.70 &
\end{tabular}

\subsection{Test Samples}

The test samples were produced with a Trumpf TruPrint 1000 Multilaser L-PBF machine. Two different sets of process parameters were used. The first set's contents are referred to as solid parameters and were used for structural parts. Samples produced with this set are from here on called solid samples. The second set is the Trumpf standard for support structure and therefore referred as support (structure) parameters. Samples using this set are henceforth called support samples. Both parameter sets use a layer height of $20 \mu \mathrm{m}$, a hatch spacing of $80 \mu \mathrm{m}$ and a laser spot size of $55 \mu \mathrm{m}$. The difference between the sets is the used energy input resulting from a laser power of $150 \mathrm{~W}$ with a scanning speed of $1600 \mathrm{~mm} \mathrm{~s}^{-1}$ as support structure parameters and $600 \mathrm{~mm} \mathrm{~s}^{-1}$ at $83 \mathrm{~W}$ for the solid samples respectively. According to Figure 1, only the solid parameters result in a fully dense part.

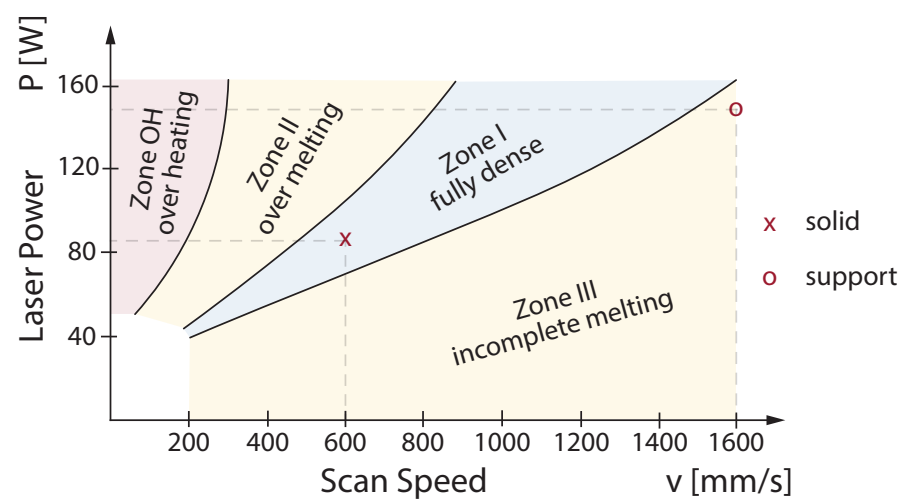

Figure 1. Process parameter relationships for L-PBF manufactured Ti-6Al-4V. Adapted from [18]. 
The samples had to be designed with a similar outline as the flat version of the ASTM E8 standard tensile test sample for metallic materials with little modifications in the dimensions to fit the clamping mechanism of the tensile machine. The middle section was replaced by the support structure that was to be tested in the tensile test. In this way, the upper and lower block of the sample acted as an aid for the clamping of the sample. A sketch of the geometry is shown in Figure 2. Bar support was used because of its similarity to the stems of tree-like support. For each set of parameters, test samples were produced in an upright orientation with a diameter $D$ range of the struts of $0.1 \mathrm{~mm}$ to $1 \mathrm{~mm}$ in $0.1 \mathrm{~mm}$ steps. The length $A$ was set to $10 \mathrm{~mm}$. The distance between the struts $L$ is the resulting value, when using four struts symmetrically with specified diameters. Samples with a diameter beneath $0.6 \mathrm{~mm}$ got heavily distorted during the printing process and were therefore not considered in this investigation. Samples with diameters above $0.7 \mathrm{~mm}$ were designed to have only three struts for consistent clearance between the struts and were therefore also excluded from this particular contribution. This leads to a remaining number of 20 test samples, five samples for each diameter and machine parameter configuration. The samples were labeled according to their sizes and diameters as L10D06 and L10D07. With respect to support structure parameters, the letter $S$ is added to the end of name for support samples.

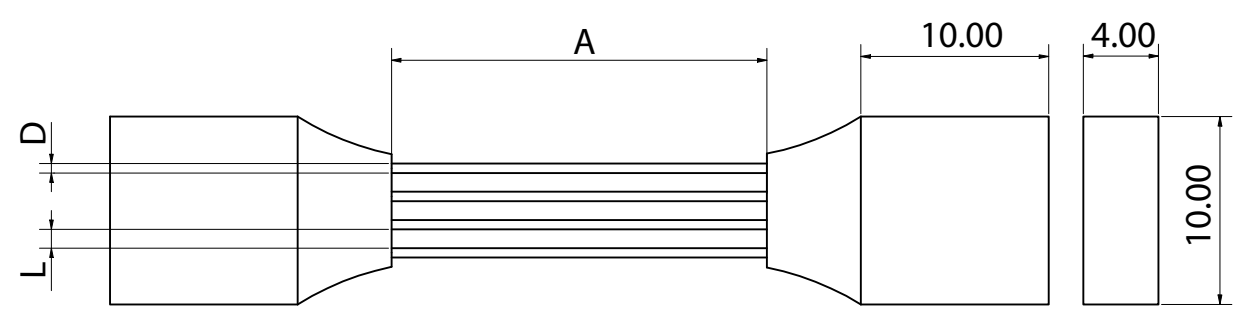

Figure 2. Sketch of the sample geometry.

\subsection{Microscopic Analyses}

The samples were analyzed using a Keyence 3D laser scanning confocal microscope of the model VK-X200. It is equipped with a violet laser with a wave length of $408 \mathrm{~nm}$. A $10 \times$ magnification objective lens was used for the measurements. The captured data have been further processed using a manufacturer provided software. The number of and sizes of defects were analyzed, as were the outer diameter and the diameter of an inner section that was present due to non-fully-molten particles. Additionally the depth information was used to create a 3D model of the fracture surface; exemplary results of this are shown in Figure 3 of Section 3.3.

After the analysis with the confocal laser microscope, the fracture surface of one sample for each parameter set was investigated using a Zeiss Ultra Plus field emission scanning electron microscope (FE-SEM), now referred to as SEM. The acceleration voltage range was $0.02 \mathrm{kV}$ to $30 \mathrm{kV}$ and was set to $1 \mathrm{kV}$ and $1.5 \mathrm{kV}$ for this contribution. A total magnification of $1,000,000 \times$ and thereby a resolution of $0.8 \mathrm{~nm}$ can be reached with this SEM. As the test samples and the desired images were rather large, the maximum magnification for this investigation was $3000 \times$.

\subsection{Micro Tensile Testing}

Tensile tests were performed on each sample of the sets in as-built condition without any mechanical post-processing other than removing the samples from the base plate. The tensile testing machine used was a tension/compression module developed and distributed by Kammrath and Weiss $\mathrm{GmbH}$ (Dortmund, Germany). The measurement range of the load cell is between $10 \mu \mathrm{N}$ and $10 \mathrm{kN}$. The machine uses threaded bars to move one part of the sample fixation with a resolution of $100 \mathrm{~nm}$ and speeds of $0.1 \mu \mathrm{m} \mathrm{s}^{-1}$ to $20 \mu \mathrm{m} \mathrm{s}^{-1}$. For this investigation a constant speed of $1 \mathrm{~mm} \mathrm{~min}^{-1}$ was used. Due to the unpredictable region of the failure on the strut and no available extensometer, the local strain of the struts was not measured. 


\section{Results}

\subsection{Geometric Accuracy of the Samples}

The diameters of the struts of the test samples were measured before and after the tensile testing. Table 2 shows the measured and the designed values for the diameter of the struts. The standard deviation $\left(\sigma_{d}\right)$ is given in parentheses. Side view is the diameter, based on the thickness of the struts, measured under the confocal laser microscope before the tensile test. Both the fracture surface and core value were measured after the test from the top view on the fracture surface with the core as the fully-molten region, which can be observed later in Figure 3. It can be recognized that the designed diameter is a little bit smaller than the actual one. After the test, the outer diameter of the fracture surface was smaller than the side view value, but still in the range of the designed diameter. Even though the difference between those diameters is in the order of the standard deviation, the reduction might result from necking due to a minor ductile behavior. Lastly the diameter of the fully dense inner section of the fracture surface was determined. This core is around the size of two powder particles smaller than the outside diameter, which results from particles being sintered or partially molten to the outside geometry due to lower heat, where laser paths no longer overlap. This is consistent with the results from the fracture surface analysis in Section 3.3, using the laser and scanning electron microscope.

Table 2. Mean values of the measured diameters $(d)$ of the test samples in $\mu \mathrm{m}$.

\begin{tabular}{lcccc}
\hline Sample & Designed Diameter & Side View & Fracture Surface & Core \\
\hline L10D06 & 600 & $606\left(\sigma_{d}=12\right)$ & $599\left(\sigma_{d}=13\right)$ & $480\left(\sigma_{d}=13\right)$ \\
L10D06S & 600 & $627\left(\sigma_{d}=7\right)$ & $607\left(\sigma_{d}=6\right)$ & $464\left(\sigma_{d}=12\right)$ \\
L10D07 & 700 & $727\left(\sigma_{d}=16\right)$ & $714\left(\sigma_{d}=8\right)$ & $565\left(\sigma_{d}=13\right)$ \\
L10D07S & 700 & $726\left(\sigma_{d}=19\right)$ & $701\left(\sigma_{d}=11\right)$ & $560\left(\sigma_{d}=14\right)$ \\
\hline
\end{tabular}

\subsection{Tensile Properties}

The ultimate tensile strength (UTS) was evaluated using the different diameters from Table 2 from before and after the tensile test to calculate the cross-sectional area and the measured forces from the micro tensile test for each sample in as-built condition. UTS side uses the diameter measured from side view, $U T S_{\text {fracture }}$ and $U T S_{\text {core }}$ respectively the outer and inner diameter measured from the top view of the fracture surface. The results are listed in Table 3. Significantly lower UTS values for the sample L10D06S with support parameters can be seen at first sight while the standard deviation is in the order of ten higher than the others. This results from a failure of some of the test samples at low stress levels without distinct plastic deformation leading to a larger standard deviation as with the other samples. Comparing the L10D07S sample with those for solid parameters no significant deviation can be observed, as the values of solid samples are only $2 \%$ to $4 \%$ higher.

Table 3. Mean ultimate tensile strength (UTS) of the as-built test samples in MPa.

\begin{tabular}{lccc}
\hline Sample & UTS $_{\text {side }}$ & UTS $_{\text {fracture }}$ & UTS $_{\text {core }}$ \\
\hline L10D06 & $852(\sigma=11)$ & $872(\sigma=12)$ & $1357(\sigma=18)$ \\
L10D06S & $640(\sigma=113)$ & $685(\sigma=119)$ & $1114(\sigma=245)$ \\
L10D07 & $839(\sigma=12)$ & $870(\sigma=13)$ & $1389(\sigma=20)$ \\
L10D07S & $808(\sigma=13)$ & $867(\sigma=14)$ & $1358(\sigma=22)$ \\
\hline
\end{tabular}

\subsection{Fracture Surface Analysis}

In Figure 3 the fracture surface images of the samples L10D07S with and L10D07 without support parameters are shown together with a 3D model of the fracture captured with a confocal laser microscope. In Figure 3a 14 defects can be observed on the fracture surface of the test sample, 
which was built using the solid parameter set. These defects seem to be pores and are investigated later in Section 3.4. The total area of the pores is around $2 \%$ with respect to the cross-sectional area. The diameters of the pores range from $7.9 \mu \mathrm{m}$ to $21.3 \mu \mathrm{m}$ with a mean diameter of $12.9 \mu \mathrm{m}$ and a standard deviation of $3.5 \mu \mathrm{m}$. All of the eight tested struts show the same number of pores. In comparison to (a), Figure $3 \mathrm{~d}$ shows the same strut of a test sample that was produced with the support parameter set. There is no visible porosity on the fracture surface of Figure $3 \mathrm{~d}$. Figure $3 \mathrm{~b}$ shows the measurement of the outside diameter of the strut from the solid sample, which is listed for all measurements of all samples in Table 2 as fracture surface. Figure $3 e$ shows the same measurement for the support sample. In both pictures an outer region with powder particles, which are not fully molten, and an inner circle with the fully dense part, the core, can be observed. The magnitude of the differences between those two diameters is in the order of two powder particles. In Figure $3 c, f$ the 3D model of the fracture is shown. It can be observed that the fracture is angled at almost $45^{\circ}$ with a slightly steeper angle in Figure 3f. This angle may result from small variations in the fixation of the samples in the tensile test machine and therefrom result in shear stresses. All in all, it still shows a predominantly brittle behavior. Especially in Figure 3f, some indications of a cup-and-cone fracture mode behavior are visible, leading to the assumption that ductility was present to some extent. The small diameters of the test samples may affect the formation of the cup-and-cone mode. The analysis of brittle and ductile behavior of the test samples will be addressed later in this article.
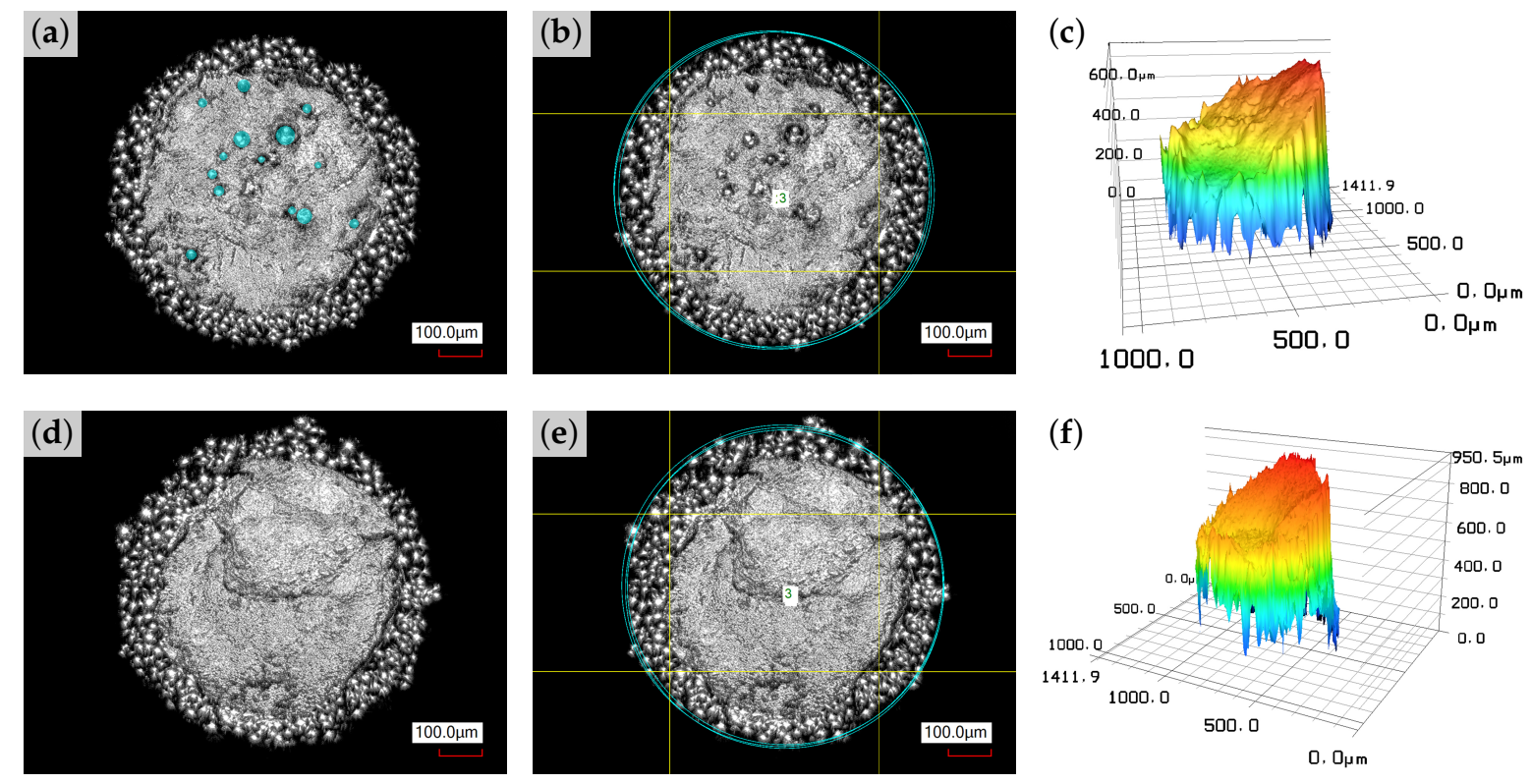

Figure 3. Confocal laser microscopy of the fracture surfaces of the test samples L10D07 (a-c) and L10D07S (d-f). Observed pores marked blue in (a), not present in (d). Outside diameter measurement of the struts in $(\mathbf{b}, \mathbf{e})$. 3D model of each fracture surface respectively in $(\mathbf{c}, \mathbf{f})$.

In Figure 4 different pictures of the fracture surface from the L10D06S sample with support parameters captured by a scanning electron microscope are displayed. At first sight, the hard edges on the fracture surface in Figure 4a indicate a brittle behavior of the fracture. Beside those edges, a single round pore can be observed in the upper half of Figure $4 \mathrm{a}$. The spheres on the outer diameter of the sample, visible in Figure $4 a, b$, are residual powder particles, which are either semi-molten or sintered to the outside surface. Especially in Figure $4 b$, it can be observed that the sizes of the particles are mostly consistent, although a few smaller particles are attached to the larger ones. A closer examination of Figure $4 \mathrm{~b}$ unravels two different types of fracture surface region, one being a brittle section with sharp edges and an obvious uneven surface with differences in height. The other region is more flat and characterized by a micro-dimpled surface that suggests a minor ductile behavior. This can be 
confirmed with Figure $4 \mathrm{c}$ with $3000 \times$ magnification. Here the micro-dimpled surface seems again to be very flat with small quasi-cleavage regions with a width of around $2 \mu \mathrm{m}$ in between the elevated grain boundaries. Bassoli and Denti refer to a similar flat micro-dimpled surface as quasi-flat rupture morphology [19]. This micro-dimpled surface combined with tiny quasi-cleavage regions suggests a small granularity of the microstructure matrix. Again, a mixture of brittle and ductile behavior can be observed in Figure 4c with the brittle region with harsh edges in the upper half of the picture and the ductile section, represented by the micro-dimpled surface, in the lower half. Several brittle sections are also visible in Figure $4 \mathrm{~d}$. The path of the edges suggests a crack initiation from a ductile outer region towards the center of the strut.
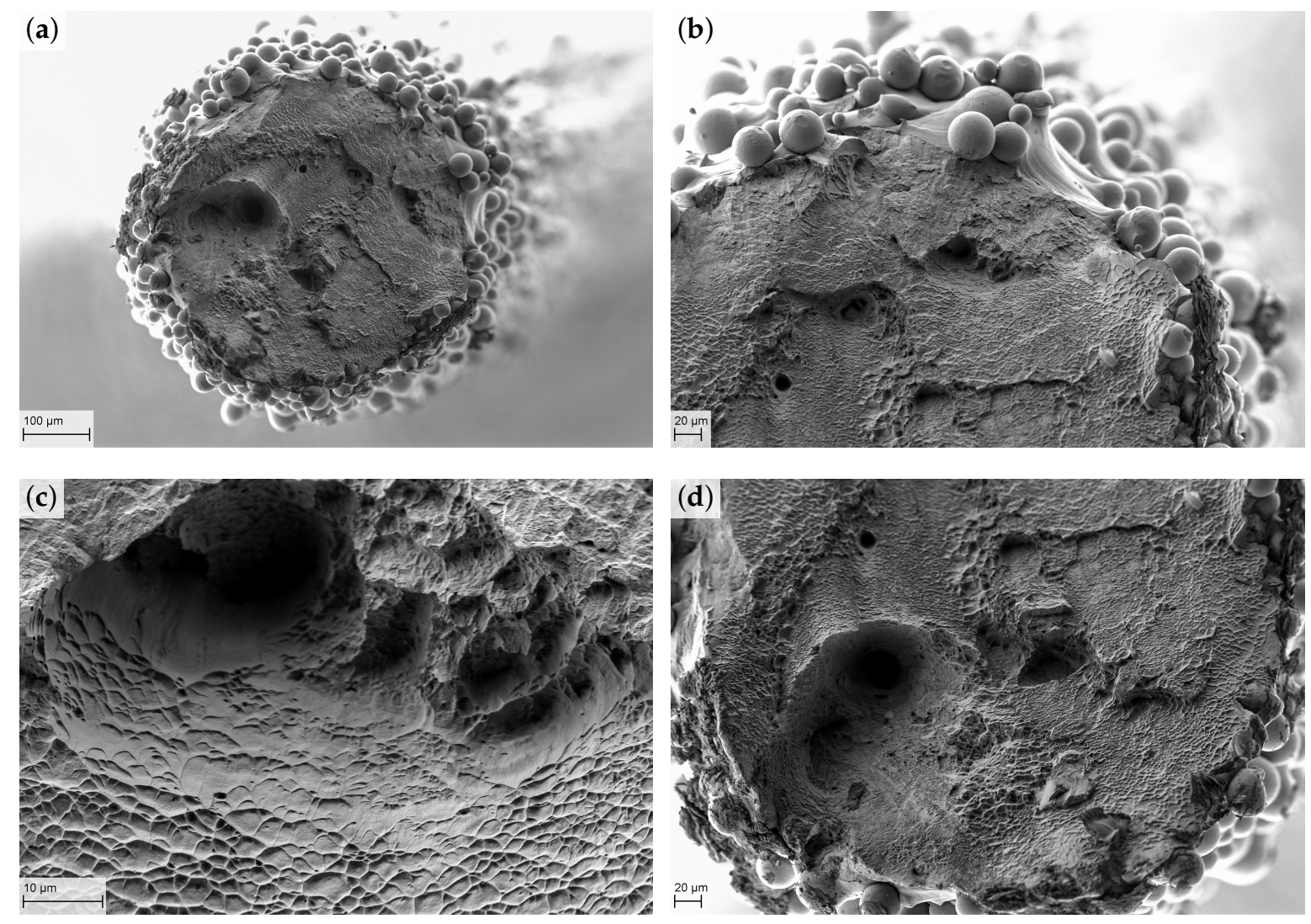

Figure 4. SEM analysis of the support sample L10D06S. (a) Fracture surface with $250 \times$ magnification. (b) Brittle and ductile regions at 500×. (c) Micro-dimpled surface at $3000 \times$. (d) Mixed fracture at 500 $\times$.

The fracture surface of the solid sample L10D06 captured with an SEM is shown in Figure 5. At first sight, there are way more defects or pores visible in Figure 5a compared to Figure 4a. Additionally, a large brittle section can be observed in Figure 5a near to the center of the strut. This brittle region is associated with the number of pores in the fracture layer. For the solid sample, there are again only a few semi-molten and sintered particles attached to the outside surface of the sample, visible in $(a, b, d)$. This shows no difference to the support sample. Brittle regions seem to evolve around pores, as can be observed in Figure 5b, even though it shows a mixed behavior close to the outside surface. Additionally, close to the outer diameter is the very flat micro-dimpled surface of Figure 5c. Around the pore a large quasi-cleavage area can be observed. Figure $5 \mathrm{~d}$ exhibits a ductile behavior in the outer region in form of a micro-dimpled surface, leading to the assumption of a small amount of necking. 


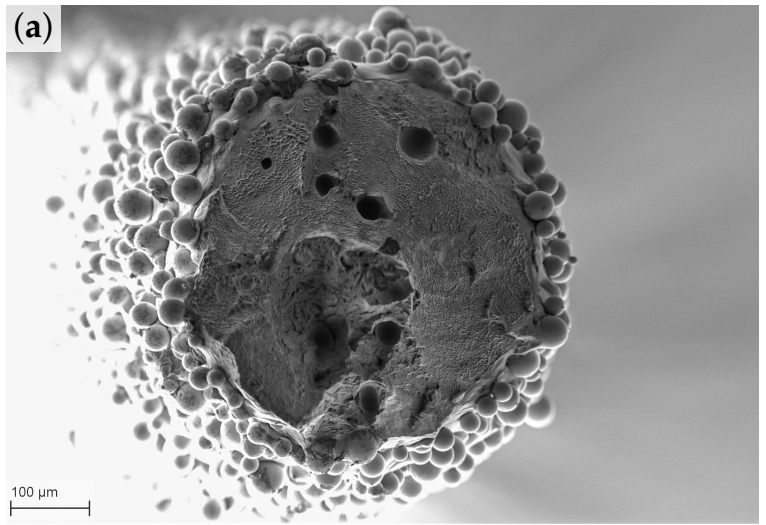

(b)
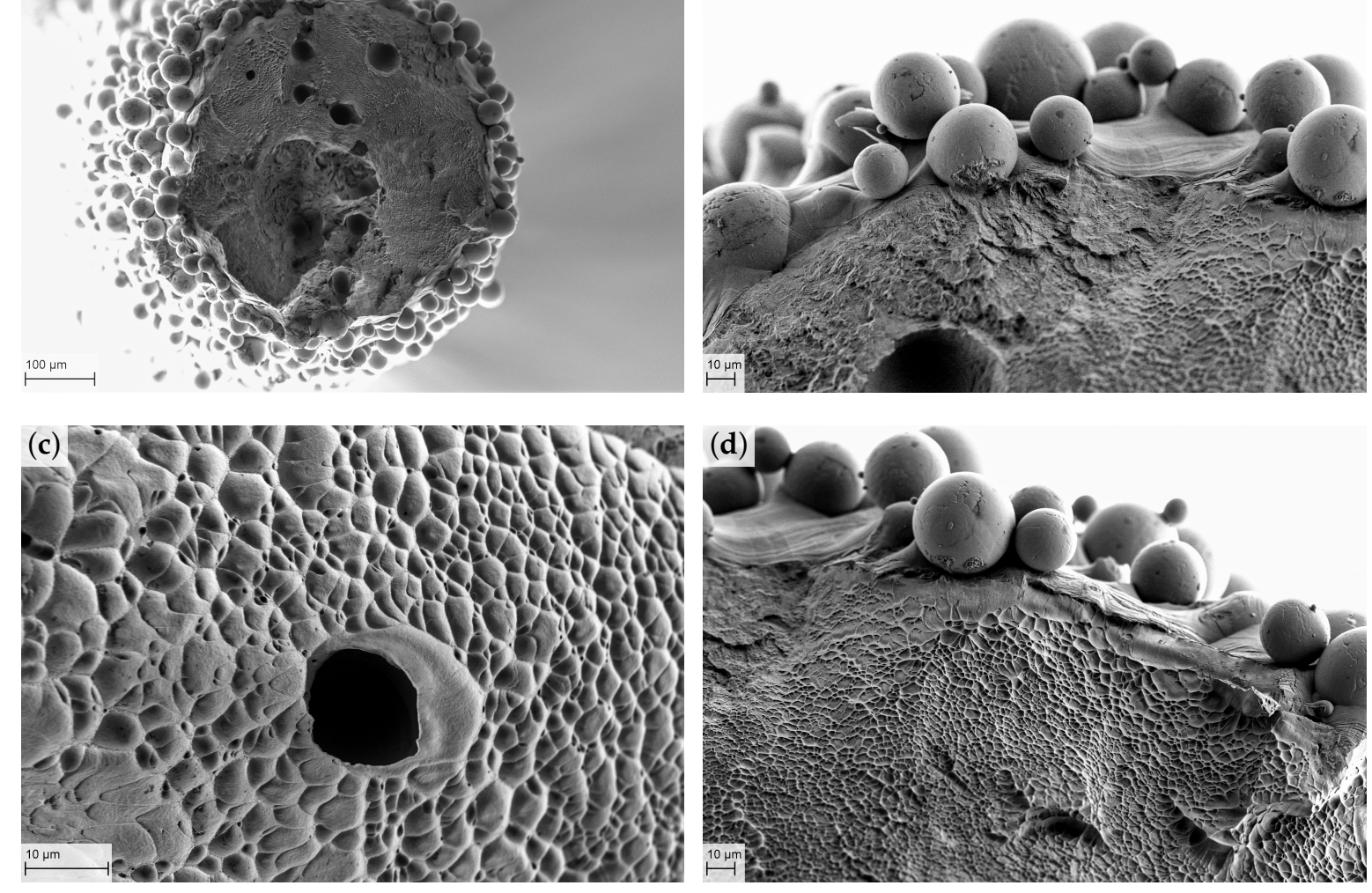

Figure 5. SEM fracture analysis of the solid sample L10D06. (a) Overview with pores at 250× magnification. (b) Mixed fracture behavior at $1000 \times$. (c) Quasi-flat dimpled surface with pore at $3000 \times$. (d) Dimpled surface close to the outside surface at $1000 \times$

During the Investigations of the fracture surface, martensite columns could be observed in regions of semi-molten particles on the outside surface. Figure 6a shows the location to be close to the edge of the fracture surface with a semi-molten particle attached to the outside surface. In Figure $6 \mathrm{~b}$ the actual martensite columns with a length of around $2 \mu \mathrm{m}$ and an approximate width of $50 \mathrm{~nm}$ can be observed in a $90^{\circ}$ pattern on the upper half of the picture and a brittle section of the fracture surface in the lower half.
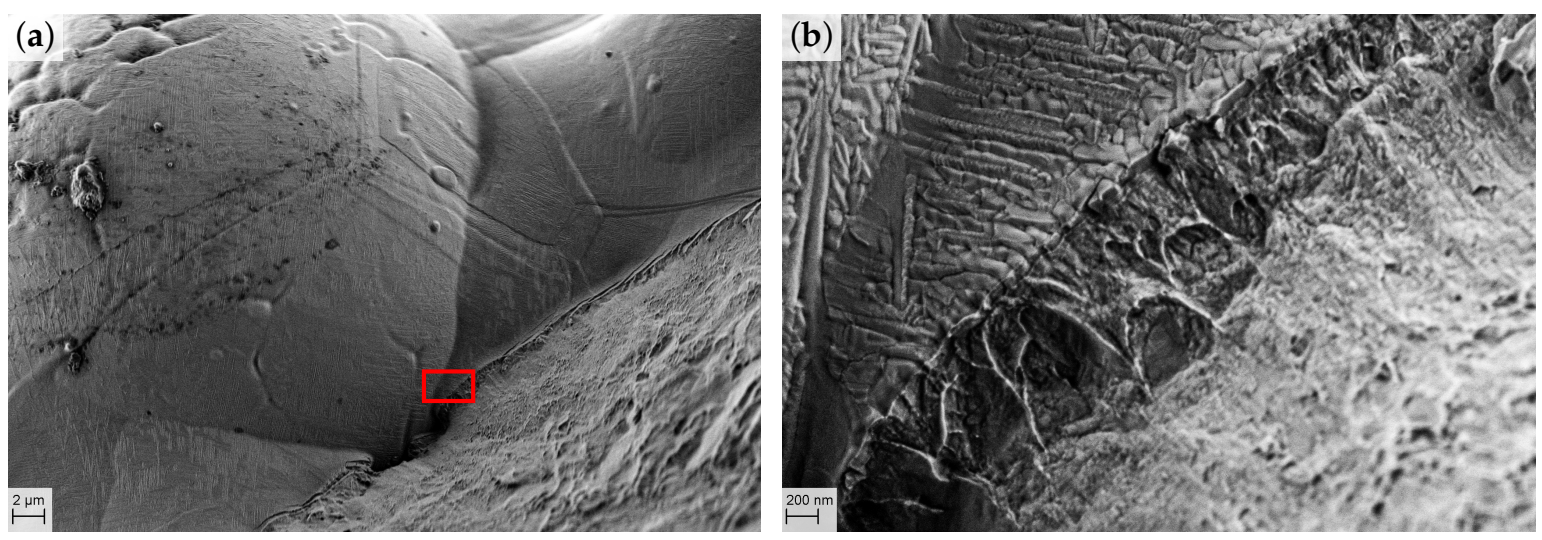

Figure 6. SEM analysis of the boundary surface of the test sample L10D06. (a) Semi-molten powder particle. (b) Magnification of (a) showing martensite columns on non fractured surface. 


\subsection{Porosity}

In order to distinguish between pores and trapped particles that fell out after the tensile test, a micro-CT scan of both samples was performed. Figure 7a shows the scan image of one half of the solid sample L10D07 through the center of the specimen. Figure 7b displays the right two struts close to the rupture. Several small pores with diameters between $10 \mu \mathrm{m}$ and $40 \mu \mathrm{m}$ can be observed. Scans of the support sample showed, as expected, no signs of pores, and they were therefore not included in this article.
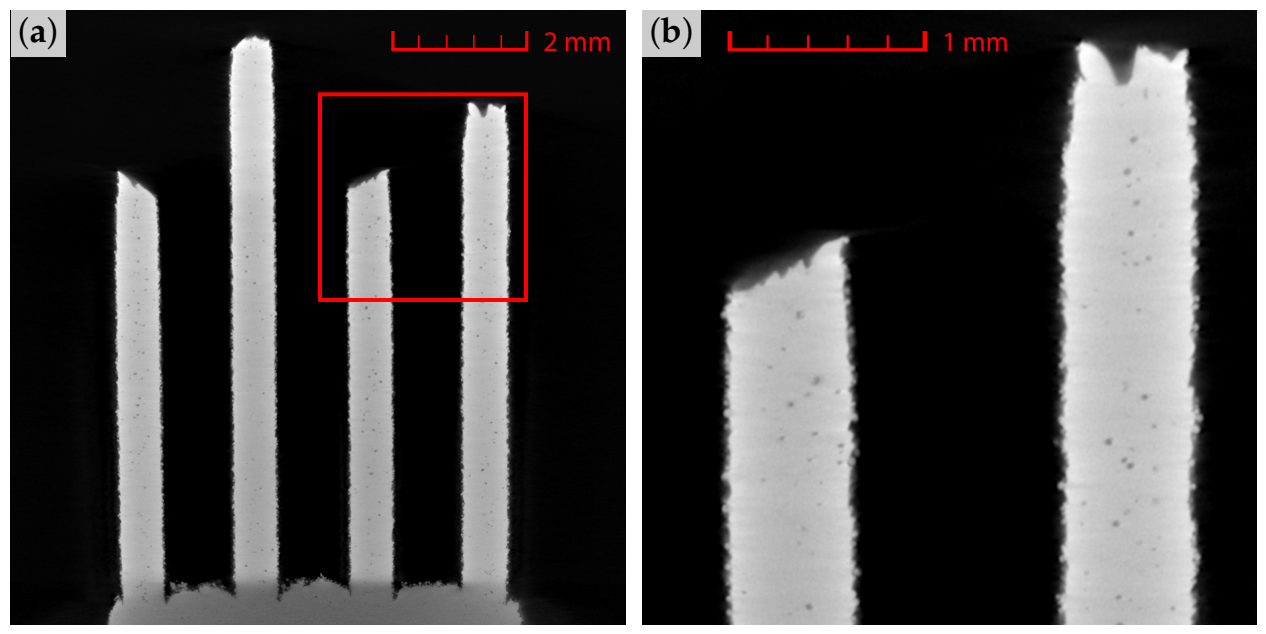

Figure 7. Micro-CT scan of the solid sample L10D07 with porosity. (a) Overview of all four struts. (b) Magnification with visible pores

Table 4 lists the mean properties of the porosity of the samples with $0.6 \mathrm{~mm}$ and $0.7 \mathrm{~mm}$ diameter struts for both parameter sets. It is clearly visible that samples produced with the solid parameter set contain more pores and thereby have larger defect surfaces. In total there is around $2 \%$ of the fracture surface subject to porosity. There is no obvious reason for the solid parameter combination to show porosity, as the combination is located in the fully dense region of Figure 1. Still, it might result from an already too high energy input or the fact that small amounts of porosity were not considered in the definition of the fully dense zone. Occasional pores in the support samples seem to have no significant differences in diameter. The sample L10D07S showed a single pore in one of the four struts. As a mean value, this leads to an amount of zero pores, but a remaining defect surface of $61 \mu^{2}$ still.

Table 4. Mean properties of pores, visible on the fracture surfaces of the test samples.

\begin{tabular}{lcccc}
\hline Sample & Number of Defects & Defect Surface $\left[\mu \mathrm{\mu m}^{2}\right]$ & Percent of Total Area & Pore Diameter $[\mu \mathrm{m}]$ \\
\hline L10D06 & 10 & $6267\left(\sigma_{s}=2075\right)$ & $2.23 \%$ & $15.1\left(\sigma_{d}=2.9\right)$ \\
L10D06S & 2 & $769\left(\sigma_{s}=2917\right)$ & $0.36 \%$ & $11.4\left(\sigma_{d}=3.3\right)$ \\
L10D07 & 12 & $6970\left(\sigma_{s}=2924\right)$ & $1.74 \%$ & $12.3\left(\sigma_{d}=3.7\right)$ \\
L10D07S & 0 & $61\left(\sigma_{s}=106\right)$ & $0.02 \%$ & - \\
\hline
\end{tabular}

\section{Discussion and Conclusions}

In this article, the characteristics of thin struts, which act as a support structure, were analyzed for two sets of build parameters. Tensile tests and subsequent fracture surface analysis were performed. It was shown that semi-molten and sintered particles on the outside surfaces of the samples affect the load bearing of the parts as the designed dimensions include these regions with particularly lower bonding strength. Small amounts of porosity in the parts showed no effect on the overall tensile strength. 
Compared to round tensile test specimens produced according to the ASTM standards, the ultimate tensile strength evaluated in this article, is significantly lower. For cross-sectional areas calculated with the outside diameters of the specimens, the UTS was below $900 \mathrm{MPa}$ and thereby strongly deviated from the range reported by Kasperovich and Hausmann given as $1040 \mathrm{MPa}$ to $1062 \mathrm{MPa}$ [20]. On the other hand, if only the core area is considered, values above $1300 \mathrm{MPa}$ are even higher than the strength of as-built and machined samples, for which Vilaro et al. evaluated $1166 \mathrm{MPa}$ [21] and Rafi et al. 1219 MPa [12]. This confirms that the outside, non-fully-molten region carries significantly less load than the core region, while still contributing to the overall load bearing. The values for L10D06S are considered statistical outliers, as the failure at low stress levels might result from a slightly tilted clamping or minor defects in the sample. The high standard deviation indicates that it was an unusual failure and if added to the UTS value it results in the same range as the other values.

The fracture surface analysis revealed a mixed fracture behavior showing micro-dimpled regions for ductile and quasi-cleavage sections for brittle failure. This is in accordance with results from Krakhmalev et al. [13] and Bassoli and Denti [19]. The cup-and-cone shape of the necking region reported by Krakhmalev et al. [13], however, was only present to some extent for the struts with small diameters. Additionally no distinct necking could be observed, as the diameter reduction was only in the range of the standard deviation of the measurements.

The results discussed in this article helped us to further understand the behavior of a support structure built with specific support parameters compared to solid parameters. Using these results, support structures can be investigated in process simulations with adapted material characteristics or be designed to meet load bearing needs. In addition, it was shown that the small diameters of the struts affected the fracture behaviors and tensile strengths of the samples. The authors suggest for future research to investigate the effect of the diameter on the micro structure, especially the differences between outside regions and the cores of the samples.

Author Contributions: Conceptualization, S.W. and J.M.; data curation, S.W. and T.S.; investigation, S.W., C.P. and T.S.; resources, C.P.; supervision, K.P.; writing-original draft, S.W. and J.M.; writing-review and editing, M.B. and K.P. All authors have read and agreed to the published version of the manuscript.

Funding: This research received no external funding.

Acknowledgments: The authors like to thank the 3D Printing Center at the Bundeswehr Research Institute for Materials, Fuels and Lubricants (WIWeB) for their assistance with the L-PBF print of the test samples and for providing the CT and SEM images.

Conflicts of Interest: The authors declare no conflict of interest.

\section{References}

1. ISO. ISO/ASTM52900-15, Terminology for Additive Manufacturing-General Principles-Terminology; Technical Report; ASTM International: West Conshohocken, PA, USA, 2015. [CrossRef]

2. 3D Printing Parts for the Aviation and Aerospace Industries. Available online: https://www.ge.com/ additive/additive-manufacturing/industries/aviation-aerospace (accessed on 13 February 2020).

3. Ponche, R.; Kerbrat, O.; Mognol, P.; Hascoet, J.Y. A novel methodology of design for Additive Manufacturing applied to Additive Laser Manufacturing process. Robot. Comput.-Integr. Manuf. 2014, 30, 389-398. [CrossRef]

4. Mercelis, P.; Kruth, J. Residual stresses in selective laser sintering and selective laser melting. Rapid Prototyp. J. 2006, 12, 254-265. [CrossRef]

5. Cloots, M.; Spierings, A.; Wegener, K. Assessing new support minimizing strategies for the additive manufacturing technology SLM. In Proceedings of the Solid Freeform Fabrication Symposium (SFF), Austin, TX, USA, 12-14 August 2013; pp. 12-14.

6. Calignano, F. Design optimization of supports for overhanging structures in aluminum and titanium alloys by selective laser melting. Mater. Des. 2014, 64, 203-213. [CrossRef] 
7. Krol, T.A.; Zaeh, M.F.; Seidel, C. Optimization of Supports in Metal-Based Additive Manufacturing by means of Finite Element Models. In Proceedings of the SFF Symposium Preceedings, Austin, TX, USA, 6-8 August 2012; pp. 707-718.

8. Strano, G.; Hao, L.; Everson, R.M.; Evans, K.E. A new approach to the design and optimisation of support structures in additive manufacturing. Int. J. Adv. Manuf. Technol. 2013, 66, 1247-1254. [CrossRef]

9. Hussein, A.; Hao, L.; Yan, C.; Everson, R.; Young, P. Advanced lattice support structures for metal additive manufacturing. J. Mater. Process. Technol. 2013, 213, 1019-1026. [CrossRef]

10. Malekipour, E.; Tovar, A.; El-Mounayri, H. Heat Conduction and Geometry Topology Optimization of Support Structure in Laser-Based Additive Manufacturing. In Mechanics of Additive and Advanced Manufacturing, Volume 9; Wang, J., Antoun, B., Brown, E., Chen, W., Chasiotis, I., Huskins-Retzlaff, E., Kramer, S., Thakre, P.R., Eds.; Springer International Publishing: Cham, Switzerland, 2018; pp. 17-27. [CrossRef]

11. Bartsch, K.; Herzog, D.; Emmelmann, C.; Lange, F. A Novel Approach to Support Structures Optimized for Heat Dissipation in SLM by Combining Process Simulation with Topology Optimization. In Proceedings of the NAFEMS World Congress 2019, Quebec City, QC, Canada, 17-20 June 2019; p. 25.

12. Rafi, H.K.; Karthik, N.V.; Gong, H.; Starr, T.L.; Stucker, B.E. Microstructures and Mechanical Properties of Ti6Al4V Parts Fabricated by Selective Laser Melting and Electron Beam Melting. J. Mater. Eng. Perform. 2013, 22, 3872-3883. [CrossRef]

13. Krakhmalev, P.; Fredriksson, G.; Yadroitsava, I.; Kazantseva, N.; Plessis, A.D.; Yadroitsev, I. Deformation Behavior and Microstructure of Ti6Al4V Manufactured by SLM. Phys. Procedia 2016, 83, 778-788. [CrossRef]

14. Simonelli, M.; Tse, Y.Y.; Tuck, C. Effect of the build orientation on the mechanical properties and fracture modes of SLM Ti-6Al-4V. Mater. Sci. Eng. A 2014, 616, 1-11. doi:10.1016/j.msea.2014.07.086. [CrossRef]

15. Popovich, A.; Sufiiarov, V.; Borisov, E.; Polozov, I. Microstructure and Mechanical Properties of Ti-6Al-4V Manufactured by SLM. Key Eng. Mater. 2015, 651-653, 677-682. [CrossRef]

16. Yadroitsev, I.; Krakhmalev, P.; Yadroitsava, I.; Du Plessis, A. Qualification of Ti6Al4V ELI Alloy Produced by Laser Powder Bed Fusion for Biomedical Applications. JOM 2018, 70, 372-377. [CrossRef]

17. Trumpf. Batch Test Report-Ti64Eli-A 17-E361; Trumpf GmbH + Co. KG: Ditzingen, Germany, 2019.

18. Gong, H.; Rafi, K.; Gu, H.; Starr, T.; Stucker, B. Analysis of defect generation in Ti-6Al-4V parts made using powder bed fusion additive manufacturing processes. Addit. Manuf. 2014, 1-4, 87-98. [CrossRef]

19. Bassoli, E.; Denti, L. Assay of Secondary Anisotropy in Additively Manufactured Alloys for Dental Applications. Materials 2018, 11, 1831. [CrossRef] [PubMed]

20. Kasperovich, G.; Hausmann, J. Improvement of fatigue resistance and ductility of TiAl6V4 processed by selective laser melting. J. Mater. Process. Technol. 2015, 220, 202-214. [CrossRef]

21. Vilaro, T.; Colin, C.; Bartout, J.D. As-Fabricated and Heat-Treated Microstructures of the Ti-6Al-4V Alloy Processed by Selective Laser Melting. Metall. Mater. Trans. A 2011, 42, 3190-3199. [CrossRef] 